\begin{tabular}{|c|c|c|c|}
\hline Eiszeitalter u. Gegenwart & $\mathbf{2 9}$ & $\begin{array}{c}23-34 \\
4 \text { Abb., } 1 \text { Tab. }\end{array}$ & Hannover 1979 \\
\hline
\end{tabular}

\title{
Die Seeablagerungen vom Samerberg/Obb. und ihre Stellung im Jungpleistozän
}

\author{
EBERHARD GRÜGER
}

Lacustrine sedimentation, lake-water level, pollen diagram, palaeoclimate, flora (forest), Upper Pleistocene (Riss, Würm) Bavarian Plateau (Samerberg), TK 25: Nr. 8139

Kurzf assung: Die Seeablagerungen vom Samerberg stammen nach dem geologischen und dem vegetationsgeschichtlichen Befund aus der Riß/Würm-Warmzeit und aus dem Frühwürm. In klimageschichtlicher Hinsicht besteht eine ausgezeichnete Übereinstimmung mit den Untersuchungsergebnissen von den ebenfalls sehr umfangreichen Profilen von Grande Pile, Rederstall, Odderade und Kittlitz, in denen über dem Eem eine durch einen Klimarückschlag zweigeteilte ältere und eine ungegliederte jüngere Waldzeit, die Frühwürm-Interstadiale Brørup und Odderade, nachgewiesen werden konnten. Diese Übereinstimmung bestätigt nicht nur die Datierung der Ablagerungen vom Samerberg, sondern erlaubt auch den Schluß, daß Eem und Riß/Würm zeitgleiche Warmzeiten sind.

\section{[The Lake Sediments of Samerberg in Upper Bavaria (Germany) and their Pleistocene Age]}

A bstract: According to the geology of the site and to the pollen analytical findings the Samerberg lake sediments are of Riss/Würmian and Early Würmian age. An excellent agreement exists between the Samerberg series and the long sequences from Grande Pile in northeastern France, Rederstall, Odderade and Kittlitz (all in northern Germany) concerning their climatic history. Here a bipartite older and a not bipartite younger forest phase, the Brørup and the Odderade interstadials, have been found above the Eemian, which is the last interglacial period in northwestern Europe. This agreement does not only confirm the dating of the Samerberg sequence, but also shows that Riss/Würmian and Eemian are same-age interglacial periods.

\section{Lage und Art des Untersuchungsgebietes}

Der Samerberg liegt am Alpennordrand südwestlich des Chiemsees im Winkel zwischen der Inntal-Autobahn und der Autobahn Rosenheim-Salzburg, die unmittelbar an seinem Fuß entlang führt. Er ist Teil eines Flyschzuges, der den nach $S$ anschließenden Kalkalpen vorgelagert ist. Während des Pleistozäns war das Gebiet mehrere Male vergletschert, zuletzt während der Würm-Kaltzeit. Pleistozäne Ablagerungen überdecken deshalb Flysch und Kalkalpin und zwar in besonderer Mächtigkeit in einer Mulde zwischen dem Flyschzug und der nördlichsten Kette der Alpen. Hier lag auch der pleistozäne See, dessen Sedimente in den letzten Jahren dank der großzügigen Unterstützung durch Herrn Max Pröbstl aus Nußdorf am Inn und seitens des Bayerischen Geologischen Landesamtes in München auf ihre pflanzlichen Einschlüsse hin untersucht werden konnten.

Im Untersuchungsgebiet treten große Höhenunterschiede auf, auf die eigens hingewiesen sei, weil sie eine höhenbedingte Zonierung der Vegetation zur Folge haben, mit der an vielen anderen Untersuchungspunkten nicht gerechnet zu werden braucht. Der Spiegel des ehemaligen Sees lag zeitweilig bei $670 \mathrm{~m}$ üb. NN, in jüngerer Zeit aber infolge Eintiefung seines Abflusses tiefer. Das nur $4 \mathrm{~km}$ entfernte Inntal und das Alpenvorland liegen etwa $450 \mathrm{~m}$ über dem Meeresspiegel. Das $3 \mathrm{~km}$ entfernte Massiv des Heubergs ist

*) Anschrift des Verfassers: Dr. E. Grüg e r, Abteilung für Palynologie der Universität Göttingen, Untere Karspüle 2, D-3400 Göttingen, Germany. 


\section{SAMERBERG $\sim 600 \mathrm{~m} \mathrm{NN}$}

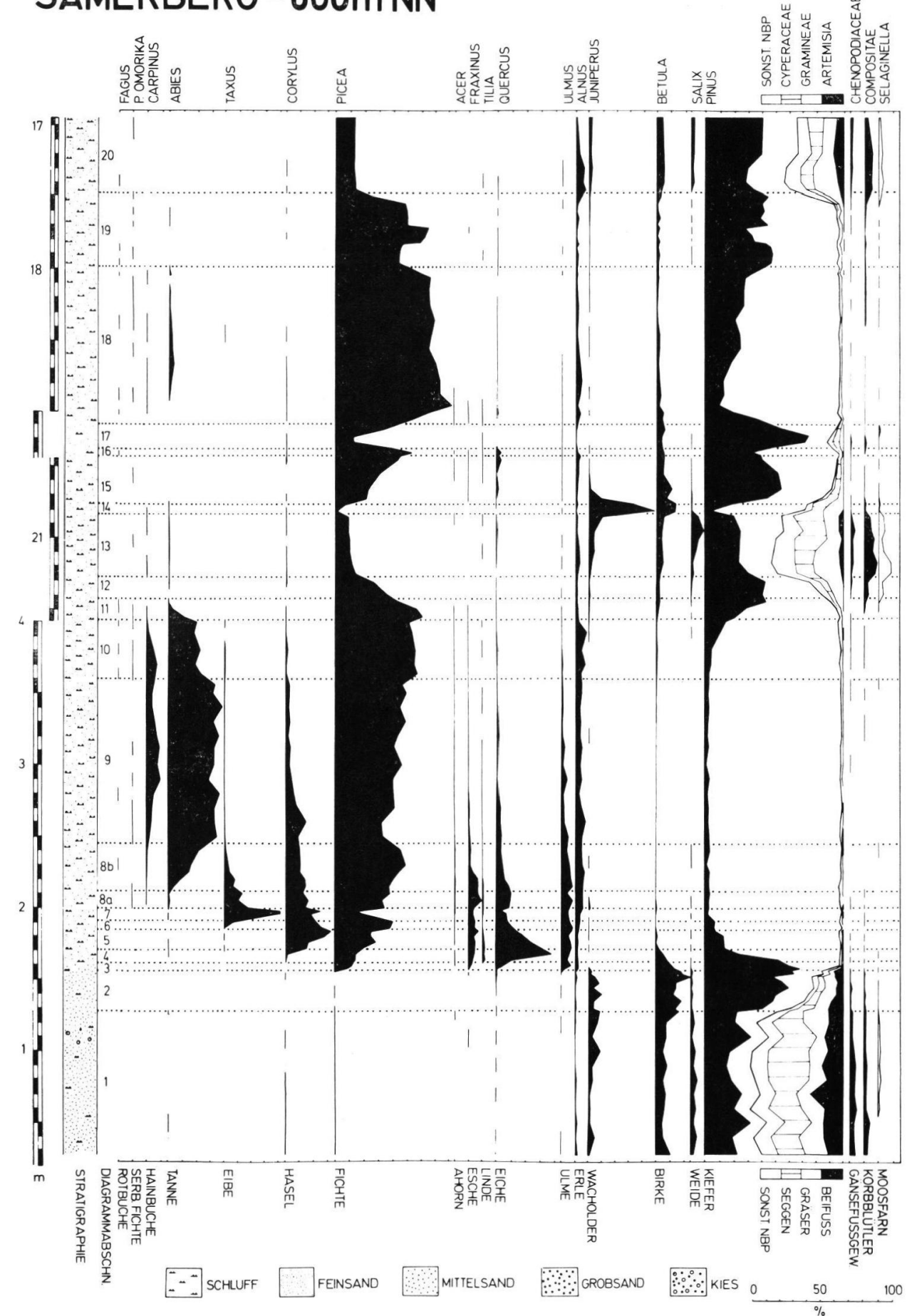

Abb. 1: Stark vereinfachtes Pollendiagramm der älteren Sedimente vom Samerberg, als Gesamtdiagramm berechnet. 


\section{SAMERBERG $-600 \mathrm{mNN}$}

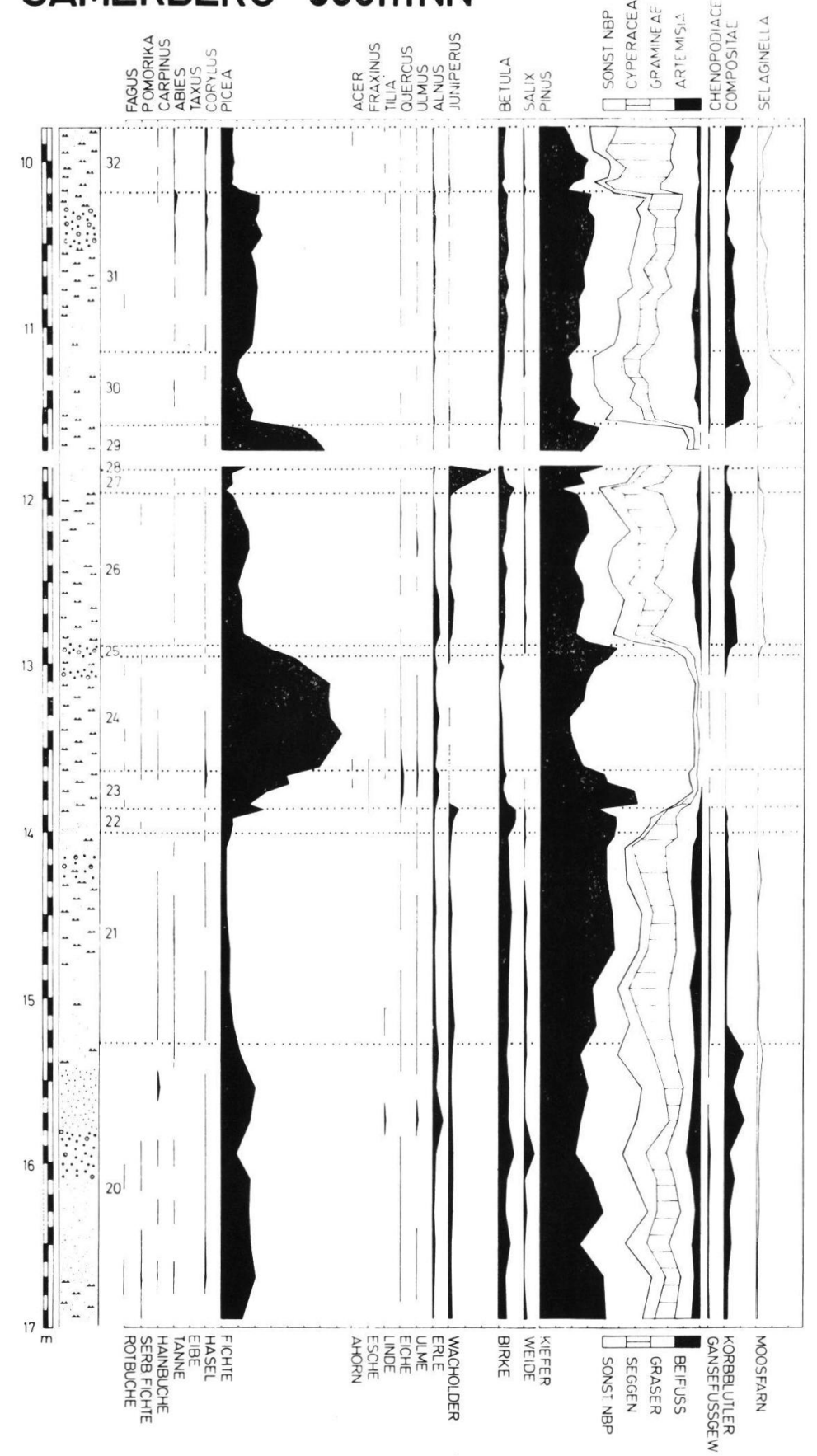

0

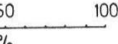

Abb. 2: Stark vereinfachtes Pollendiagramm der jüngeren Sedimente vom Samerberg, als Gesamtdiagramm berechnet. 
$1338 \mathrm{~m}$ hoch, und in $3,5 \mathrm{~km}$ Entfernung liegt die mit $1569 \mathrm{~m}$ noch höhere Hochries. Das bedeutet, daß auf etwa $7 \mathrm{~km}$ horizontale Distanz ein Höhenunterschied von $1100 \mathrm{~m}$ besteht. Entsprechend vielgestaltig ist - und war wohl auch während älterer Warmzeiten die vertikale Gliederung der Vegetation. Sie reicht heute vom Buchenwald in den tiefen und Laubmischwald in den mittleren Lagen bis zur Baumgrenze, die an der Hochries erreicht und dort von der Fichte gebildet wird.

Die Sedimente des ehemaligen Sees sind z. T. aufgeschlossen, z. T. sind sie nur in Bohrungen zugänglich. Eine Kernbohrung deckte die folgende Schichtenfolge auf (R 4515 170, H 5290 330, 612 m üb. NN, Einzelheiten bei GRüGER, im Druck):

$\begin{array}{rrrl}0-3,3 & \mathrm{~m} & \text { Oberboden und Würm-Grundmoräne } \\ 3,3-22,5 & \mathrm{~m} & \text { Seesedimente } \\ 22,5-23,8 \mathrm{~m} & \text { Grundmoräne? } \\ 23,8-37,2 \mathrm{~m} & \text { Seesedimente } \\ 37,2-37,23 \mathrm{~m} & \text { "Schieferkohle“ } \\ 37,23-37,6 \mathrm{~m} & \text { Grundmoräne. }\end{array}$

Nur die Sedimente aus 4,1 bis 22,35 m Tiefe enthielten Pollen.

\section{Die Vegetationsentwicklung}

In den Pollenspektren des ältesten im Pollendiagramm dargestellten Abschnitts (DA 1, Abb. 1) herrscht Nichtbaumpollen vor. Es handelt sich dabei vor allem um Pollen von Artemisia (Beifuß), Gramineae (Gräser), Cyperaceae (Seggen), Chenopodiaceae (Gänsefußgewächse) und Compositae (Korbblütler). Auch Selaginella selaginoides (Moosfarn) ist regelmäßig nachgewiesen. Der Gehölzpollen stammt überwiegend von Pinus (Kiefer). Daneben erreichen nur Salix (Weide), Betula (Birke) und Juniperus (Wacholder) höhere Werte. Zusammen mit Nachweisen einiger ausgesprochen lichtbedürftiger Taxa wie Ephedra (Meerträubel), Hippophae (Sanddorn), Helianthemum (Sonnenröschen) u. a. ergibt sich daraus das Bild einer offenen, möglicherweise noch völlig baumfreien Vegetation.

Birke und Kiefer leiteten die Wiederbewaldung ein (DA 2). Die Anwesenheit beider Gattungen im Gebiet ist außer durch Pollen auch durch Früchte bzw. Nadeln belegt.

Es folgte die Ausbreitung von Laubgehölzen wie Quercus (Eiche), Fraxinus (Esche), Corylus (Hasel) u. a. sowie von Picea (Fichte). Dabei erreichen Quercus bzw. die gewöhnlich zum Eichenmischwald (EMW) gerechneten Arten zusammen Höchstwerte von 38 bzw. $50 \%$ bei gleichzeitig bis zu $25 \%$ Picea-Pollen. Von Picea gibt es übrigens schon vom DA 3 an vereinzelt Nadelfunde. Die Hasel erreicht bei der hier gewählten Berechnungsweise als Gesamtdiagramm $35 \%$. Bezieht man ihre Anteile - wie bei W'aldzeiten üblich - auf die Baumpollensumme, so beträgt ihr Höchstwert $60 \%$.

Ohne auf Einzelheiten der Vegetationsentwicklung näher einzugehen, die andernorts (GrüGER, im Druck) ausführlich dargestellt werden sollen, seien im Folgenden nur die wichtigsten Veränderungen der Vegetation genannt: eine kurze, aber markante Eibenzeit (Taxus: $53 \%$, bezogen auf Baumpollen ohne Corylus 66,5\%) und die Ausbreitung von Tanne (Abies) und Hainbuche (Carpinus), die offenbar zum fast völligen Verschwinden der früher eingewanderten Laubholzarten aus der Höhenstufe des Samerberges geführt haben.

Das Ende der Waldzeit, bei der es sich - der Höhe der erreichten Vegetationsentwicklung nach - nur um eine interglaziale handeln kann, kündigt sich schließlich im DA 12 durch ein Ansteigen der Pinus-Anteile und entsprechend niedrigere Werte bei den anderen Arten an. 
Eine waldfreie Zeit (DA 13) schließt sich an. Sie weist eine ganz ähnliche Pollenflora auf wie die waldlose Zeit, welche dem Interglazial vorausging.

Danach kam es im Untersuchungsgebiet noch einmal zur Bildung geschlossener Wälder. Diese Waldzeit begann mit einer Juniperus-Phase und führte zunächst zu einer Bewaldung mit Pinus und Picea (DA 14-16).

Besonders wichtig, vor allem in Hinblick auf die Datierung sind die folgenden Veränderungen (DA 17), nämlich ein kräftiger Rückgang der Picea-Werte, das Aussetzen fast aller Kurven von Laubbaumarten, der starke Anstieg der Kiefernkurve und die erneut höheren Nichtbaumpollenanteile, an denen auch wieder Artemisia beteiligt ist.

Diese Veränderungen müssen als Anzeichen eines Klimarückschlags gedeutet werden. Durch ihn wurde die Vegetationsentwicklung - vorübergehend! - wieder auf die Stufe des Kiefernwaldes zurückgeworfen. Eine gewisse Zeit nach diesem Rückschlag konnte sich die Fichte wieder ausbreiten. Auch die Tanne erschien jetzt im Gebiet, blieb aber relativ bedeutungslos. Auch diese Waldzeit, in der anspruchsvollere Laubbaumarten in der Höhenstufe des Samerbergs wahrscheinlich ganz fehlten, endete mit einer Kiefernzeit.

Da der Klimarückschlag des DA 17 nicht zur Waldlosigkeit führte, kann man die gesamte Vegetationsentwicklung, wie sie in den DA 14-19 zum Ausdruck kommt, als eine Einheit auffassen. Es liegt also ein einziges, allerdings zweigeteiltes Interstadial vor.

Im weiteren Verlauf der Verlandung des Sees kamen zeitweise Sande und Kiese zur Ablagerung, was auf offene Vegetation oder sogar auf Flächen ohne Pflanzendecke schließen läßt, ganz in Übereinstimmung mit den Aussagen des Pollendiagramms (DA 20-21, Abb. 2).

Es folgt eine weitere Waldzeit interstadialen Charakters (DA 22-25), während der es wiederum zur Ausbildung von Fichtenwäldern kam. Dieses Mal waren am Samerberg aber nicht nur die Laubbaumarten nicht mehr vorhanden, sondern auch die Tanne scheint gefehlt zu haben.

Der DA 26 zeigt das schon bekannte Bild einer waldlosen Vegetation, und in den DA 27-29 ist ein weiteres Interstadial angedeutet, das aber nur fragmentarisch überliefert ist. Seine Vegetationsentwicklung führte wenigstens bis zur Bildung eines Fichtenwaldes. Alle jüngeren Abschnitte haben stadialen Charakter.

Insgesamt lassen sich also am Samerberg ein Interglazial im Liegenden und drei Interstadiale nachweisen, von denen das älteste durch einen Klimarückschlag gekennzeichnet ist.

\section{Die Datierung}

Die Datierung von Veränderungen der Vegetation ist auf verschiedene Weisen möglich. In günstigen Fällen geben die geologischen Verhältnisse eine klare Antwort auf die Frage nach dem Alter einer Waldzeit. Meistens muß man aber die Vegetationsentwicklung im Untersuchungsgebiet mit den entsprechenden Befunden von anderen Untersuchungspunkten vergleichen, d. h. man muß die neuen Untersuchungsergebnisse in eine bereits bestehende Rekonstruktion einer großräumigen Vegetationszonierung einordnen. Dies ist die in der Vegetationsgeschichte am häufigsten angewandte Datierungsmethode, die sich bewährt hat. Darüber hinaus kann man eine Datierung auch auf klimageschichtliche Befunde gründen. Voraussetzung dafür ist, daß ausreichend lange Pollendiagramme, d. h. solche, die mehrere Waldzeiten umfassen, zum Vergleich zur Verfügung stehen. Alle drei Datierungsmethoden sollen im Folgenden auf die pollenanalytischen Ergebnisse angewandt werden. Am detailliertesten sei aber auf die zuletzt genannte eingegangen, weil sie eine großräumige Betrachtungsweise erlaubt und am wenigsten von lokalen Besonderheiten der geologischen oder botanischen Befunde beeinflußt wird. 
Der geologische Befund legt eine Zuordnung der interglazialen Serie vom Samerberg zur Riß/Würm-Warmzeit und der kaltzeitlichen Sedimente zum Frühwürm nahe. Diese Datierung setzt voraus, daß wenigstens die jüngste der beiden Moränen im Liegenden des Interglazials eine Riß-Moräne ist, was in der Tat als das Wahrscheinlichste angenommen werden darf, letztlich aber nicht bewiesen ist.

Die Datierung mit Hilfe vegetationsgeschichtlicher Kriterien kann sich auf die Frage beschränken, ob das nachgewiesene Interglazial die Riß/Würm- oder die Mindel/RißWarmzeit darstellt, denn ein cromerzeitliches, altpleistozänes oder gar tertiäres Alter der Sedimente scheidet völlig aus, weil keine der für diese Zeiten typischen Arten am Samerberg nachgewiesen werden konnte.

Vergleicht man die Vegetationsentwicklung des Interglazials vom Samerberg mit der Vegetationsentwicklung der beiden letzten Warmzeiten in den nordwesteuropäischen und nordpolnischen Tieflandgebieten und den wenigen aus den Mittelgebirgen bekannten Abfolgen, so kommt man zu folgendem Ergebnis: eem- bzw. riß/würmzeitliche Merkmale sind am Samerberg die klare Gliederung der Vegetationsentwicklung in mehrere Abschnitte und die trotz der Anwesenheit der Fichte hohen EMW-Werte (50\%). Die Haselwerte $(60 \%)$ sind, verglichen mit den Anteilen, welche an den meisten letztinterglazialen Untersuchungspunkten erreicht werden, niedrig; für holstein- bzw. mindel/rißzeitliche Verhältnisse sind sie zu hoch.

Nicht nachweisbar ist in den Pollendiagrammen vom Samerberg der für das nordwesteuropäische und nordpolnische Eem charakteristische lindenreiche Abschnitt der EMWHaselzeit. Er fehlt aber allen bis jetzt publizierten Pollendiagrammen dieser Warmzeit aus dem Bereich der Mittelgebirge, wenn man von Wallensen in der Ith-Hils-Mulde (RABIEN 1953), das seiner Lage nach noch fast zu den Tieflandlokalitäten gerechnet werden kann, einmal absieht. Aus dem südlichen Polen sind aber Pollendiagramme bekannt, die zwischen beiden Diagrammtypen vermitteln.

Die Eibe, die am Samerberg zeitweise eine bedeutende Rolle spielte, war sowohl während der letzten als auch während der vorletzten Warmzeit am Aufbau der Wälder beteiligt. Jedoch ist eine Eibenzeit, die wie am Samerberg noch vor der Ausbildung einer Schattholzphase endet, also in einer sehr charakteristischen pollenstratigraphischen Lage angetroffen wird, bisher nur von letztinterglazialen Untersuchungspunkten aus dem Alpenvorland bekannt geworden.

Für eine Zuordnung der warmzeitlichen Sedimente vom Samerberg zum vorletzten Interglazial sprechen allenfalls das frühe und andauernde Vorkommen der Fichte, die hohen Tannenwerte und die geringe Beteiligung der Hainbuche. Diese Merkmale können aber auch Ausdruck einer Höhenstufung sein, wie sie sich unter natürlichen Bedingungen $\mathrm{zu}$ jeder Zeit in einer Gebirgslandschaft einstellen wird. Gegen eine Zuordnung zum vorletzten Interglazial spricht auch die Höhe der EMW- und Corylus-Werte.

Aus all dem kann man schließen, daß am Samerberg das Riß/Würm-Interglazial und infolgedessen auch das Frühwürm mit seinen Interstadialen nachgewiesen werden konnten.

Dieser Beweisführung soll eine dritte an die Seite gestellt werden. Sie basiert auf dem Vergleich der Klimaentwicklung, deren große Veränderungen an Pollendiagrammen abgelesen werden können, wenn diese nur lang genug sind, also mehrere W'aldzeiten umfassen. Derartig umfangreiche, ungestörte Profile sind überaus selten. Im Folgenden sollen die vier in Frage kommenden Pollendiagramme von mitteleuropäischen Untersuchungspunkten vorgestellt und dann zur Datierung der Sequenz vom Samerberg herangezogen werden. 


\section{Grande Pile}

Von Grande Pile am Südwestfuß der Vogesen hat WoIllard $(1975,1978)$ Pollendiagramme beschrieben, die mehrere durch waldlose Abschnitte voneinander getrennte Waldzeiten umfassen. Der ältesten Waldzeit (Abb. 3) geht ein spätglazialer, durch NBPDominanz gekennzeichneter, also waldloser Abschnitt voraus (DA 1). Die Wiederbewaldung führte über Abschnitte mit Juniperus-, Betula- und Pinus-Dominanz zu einer Zeit der Vorherrschaft von Quercus, Corylus und anderen Laubhölzern. Die Eibe erreichte zeitweise hohe Werte. Später wanderten Carpinus, Abies und zuletzt Picea ein und verdrängten die vorher dominierenden Laubbaumarten. Mit einer Kiefernzeit endete diese älteste interglaziale Waldzeit. Sie wird von WoIllard dem Eem zugerechnet.

Es folgt ein waldloser Abschnitt (DA 13), wiederum mit relativ hohen Artemisiaund Chenopodiaceaen-Anteilen.

Die nachfolgende Waldzeit (DA 14-16) begann ähnlich wie das Eem im Liegenden mit einer Ausbreitung von Juniperus, Betula und später auch Pinus. Sogar Quercus, Corylus, Carpinus und Picea konnten sich ausbreiten, bevor ein Klimarückschlag (DA 17) der Kiefer und krautreichen Pflanzengesellschaften vorübergehend wieder zur Vorherrschaft verhalfen. Auf diese wichtige, für anspruchsvolle Baumarten offenbar ungünstige Phase folgte die erneute und endgültige Ausbreitung dieser Arten, die hier nicht näher beschrieben zu werden braucht (DA 18-19). Wieder schließt eine Kiefernzeit den Entwicklungszyklus ab.

WOILlard (1975) bezeichnete diese durch einen Klimarückschlag in zwei Abschnitte geteilte Waldzeit als ein Interglazial und gab ihm den Namen St. Germain I. Während dieser Waldzeit traten einige der während der Eem-Warmzeit wichtigen Arten wie Abies, Taxus und Buxus fast nicht in Erscheinung, und auch die Laubbaumarten erlangten nicht mehr die Bedeutung, die sie während der Eem-Warmzeit hatten. Dies läßt vermuten, daß die klimatischen Bedingungen während dieser jüngeren Waldzeit nicht mehr so günstig waren wie während des Eems.

Eine weitere waldlose Zeit (DA 20-21, Abb. 4) leitet zu einer dritten Waldzeit, St. Germain II (DA 22-25) über, die bei gleichem Arteninventar wie während St. Germain I eine geringere Beteiligung von Carpinus und Picea aufweist, aber nicht zweigeteilt ist.

Die jüngeren Sedimente enthalten eine Pollenflora stadialen Charakters und in den DA 27-29 vielleicht ein weiteres Interstadial, über das aber noch keine endgültige Klarheit besteht, weil es sich nicht in allen Paralleldiagrammen abzeichnet.

Bei Grande Pile sind also drei aufeinanderfolgende Waldzeiten festgestellt worden, von denen die älteste die artenreichste und anspruchsvollste Flora besais und dem Eem zugerechnet wird. Von den beiden jüngeren Waldzeiten ist die ältere, St. Germain I, durch einen Klimarückschlag in charakteristischer Weise zweigeteilt.

\section{Rederstall}

Eine ganz ähnliche Abfolge fand Menke (1976) bei Rederstall in Westholstein (Tab. 1). Über eemzeitlichen Ablagerungen konnten hier die beiden Frühwürm-Interstadiale Brørup und Odderade und innerhalb des Brørup-Interstadials eine Klimaverschlechterung (WF $\left.\mathrm{II}_{2}\right)$ festgestellt werden. Sie unterbrach die beginnende Ausbreitung der Kiefer und ist im Pollendiagramm durch hohe NBP- und Juniperus-Werte markiert. Auch ArtemisiaPollen ist in diesem Abschnitt wieder verzeichnet. Höhepunkt der folgenden Vegetations- 
GRANDE PILE $X, 330 \mathrm{~m}$

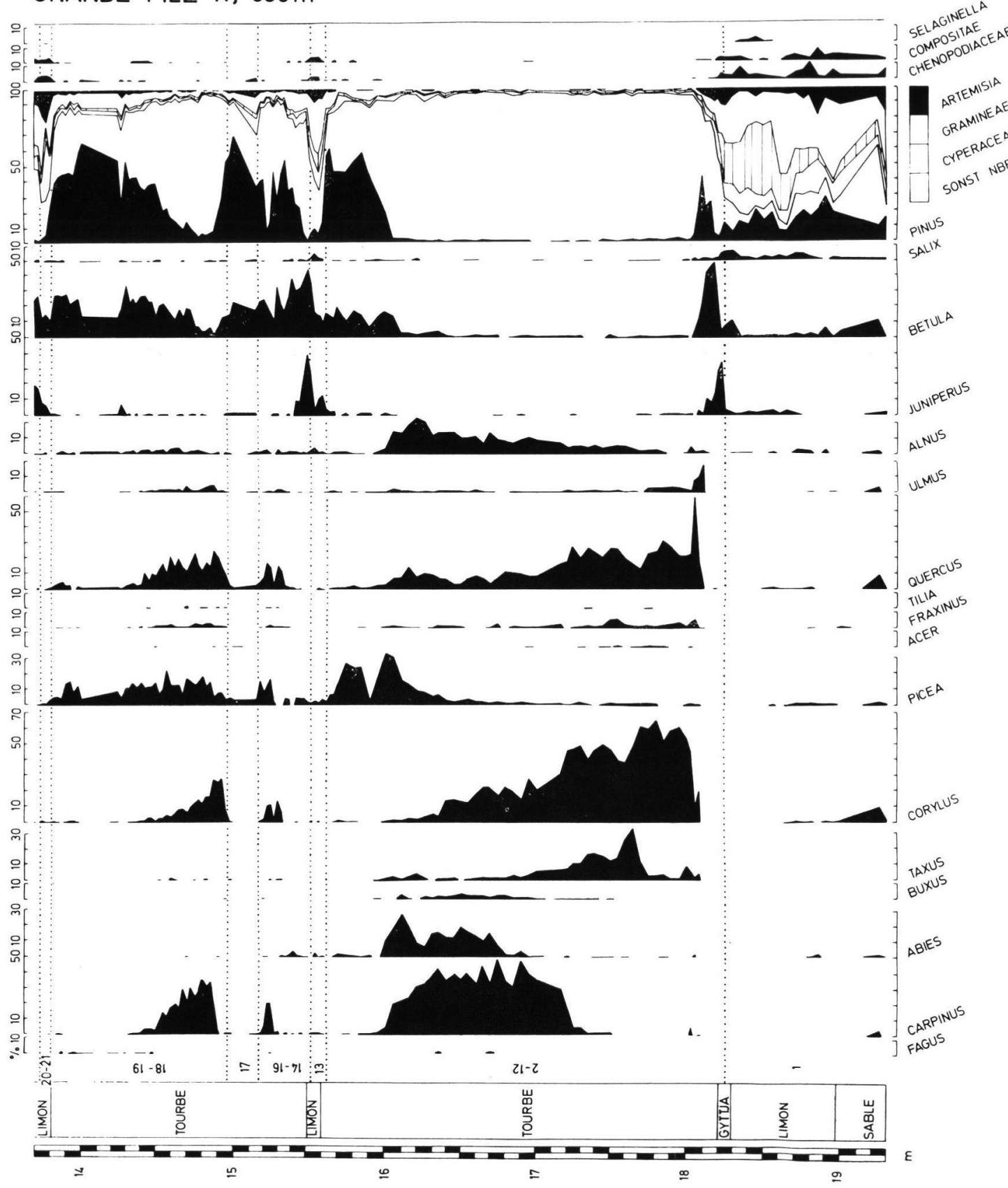

Abb. 3: Stark vereinfachtes Pollendiagramm von Grande Pile (= ältere Abschnitte des Diagramms X, WoIllard 1975), umgezeichnet und mit der Gliederung des Samerberg-Diagramms versehen. 
GRANDE PILE $X, 330 \mathrm{~m}$

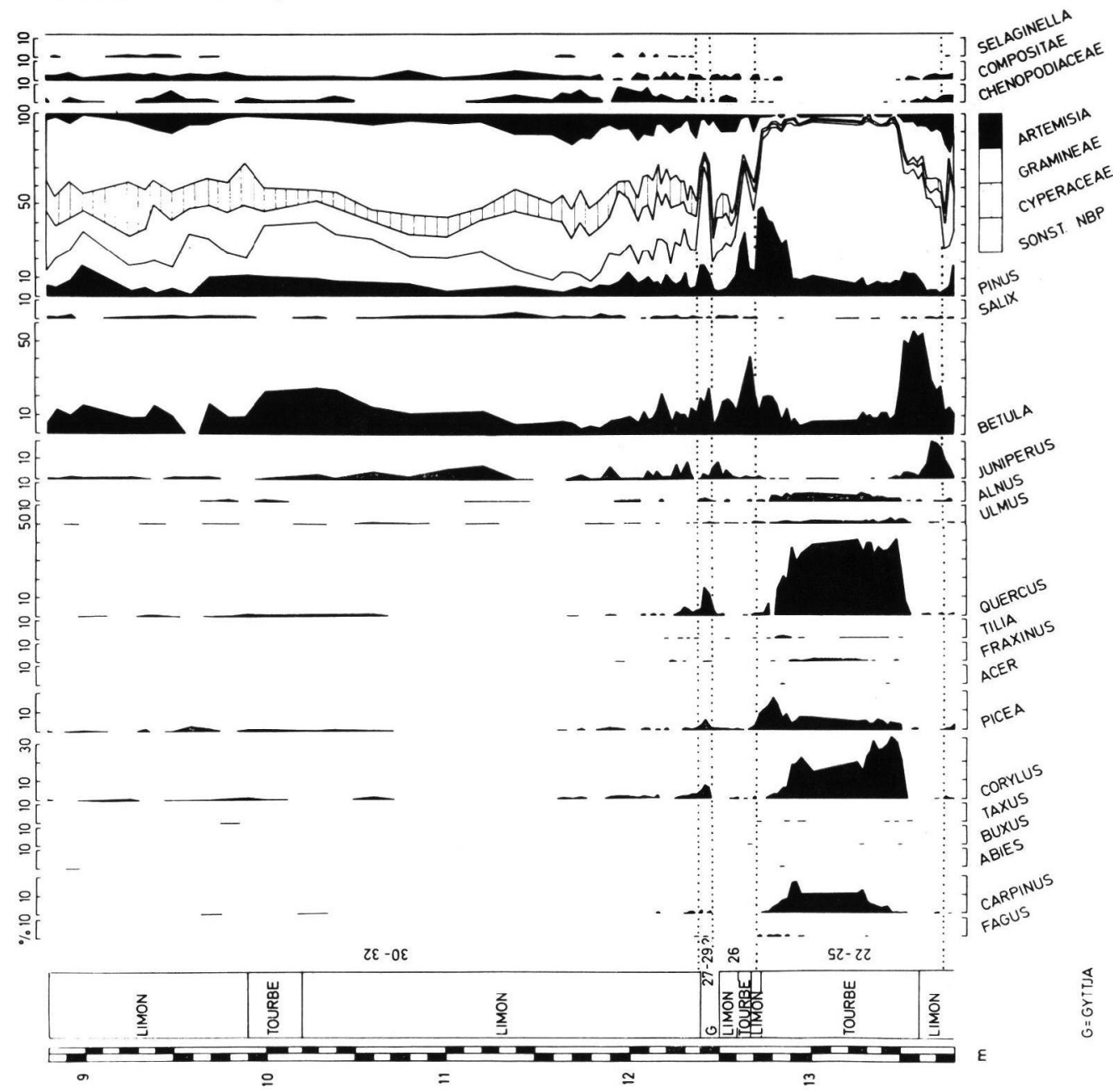

Abb. 4: Stark vereinfachtes Pollendiagramm von Grande Pile (= mittlere Abschnitte des Diagramms X, WolllaRD 1975), umgezeichnet und mit der Gliederung des Samerberg-Diagramms versehen.

entwicklung war die Bildung von Pinus-Wäldern, in denen zeitweise Larix und Picea von Bedeutung waren. (Ich danke Herrn Dr. B. Menke/Kiel sehr dafür, diese dem noch unveröffentlichten Pollendiagramm entnommenen Daten verwenden zu dürfen.)

\section{I . O d d e r a d e}

Ganz ähnliche Verhältnisse zeigt das Pollendiagramm 5 von Odderade (Averdieck 1967). Hier, an der Typus-Lokalität des Odderade-Interstadials, folgen auf die EemWarmzeit zwei "große“ Interstadiale. Dem älteren, dem Brørup-Interstadial sensu Averdieck, gehen in pollenstratigraphisch gleicher Lage wie im Pollendiagramm Rederstall und auch in ganz ähnlicherAusbildung ein wenig intensiver Klimarückschlag (FW 3) 


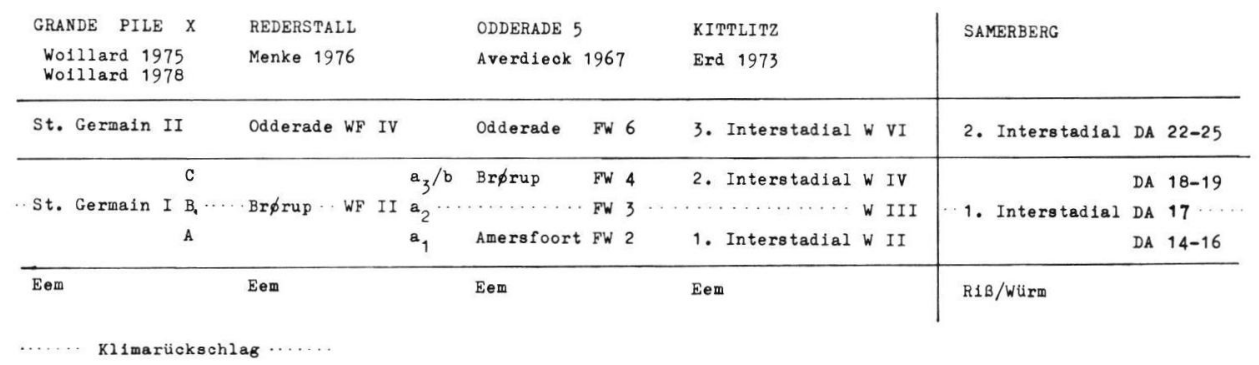

Tab. 1: Gleichalte Waldzeiten in langen Profilen aus Nordost-Frankreich, Nord- und Süddeutschland. Die Punktierung hebt den durch einen Klimarückschlag gekennzeichneten Abschnitt der ältesten frühwürmzeitlichen Waldzeit hervor.

und das Amersfoort Interstadial (FW 2), das durch Betula-Vorherrschaft gekennzeichnet ist, voraus. Schon Schneekloth (1966) stellte die Frage, ob der Abschnitt FW 2, das Amersfoort-Interstadial, in Odderade überhaupt als selbständiges Interstadial zu betrachten ist oder nicht besser - wie auch Menke (1976) vorschlug — als Teil eines zweigeteilten Interstadials angesehen werden sollte (vgl. Tab. 1).

\section{Kittlitz}

Ahnlich liegen auch die Verhältnisse bei Kittlitz in der Niederlausitz (ERD 1973). Auch hier liegen über eemzeitlichen Sedimenten solche von Frühwürm-Interstadialen und -Stadialen. Auch hier folgte auf das älteste sehr schwach entwickelte Interstadial (W II) ein vergleichsweise wenig intensiver Klimarückschlag (W III), und erst danach kam es zur Bildung von Kiefernwäldern mit Fichte (W IV). Ein weiteres, ungegliedertes Interstadial (W VI) folgte nach einer waldlosen Phase (vgl. Tab. 1).

Das gleiche Alter der drei zuletzt beschriebenen Abfolgen - Eem-Warmzeit/zweigeteiltes Brørup-Interstadial/ungeteiltes Odderade-Interstadial - ist erwiesen. Die älteste Waldzeit von Grande Pile gehört, wie Wolllard (1975) sicherlich mit Recht feststellte, der Eem-Warmzeit an. Angesichts der übereinstimmenden weiteren Klimaentwicklung müssen die übrigen Waldzeiten, St. Germain I und II, zeitlich den Frühwürm-Interstadialen entsprechen.

Wenden wir uns wieder den Verhältnissen am Samerberg zu. Hier konnten ein Interglazial und darüber Interstadiale, von denen das älteste in charakteristischer Weise zweigeteilt ist, nachgewiesen werden. Offensichtlich ist diese Abfolge mit den Sequenzen von Grande Pile, Rederstall, Odderade und Kittlitz gleichzusetzen, mit denen sie in klimageschichtlicher Hinsicht völlig übereinstimmt. Demnach sind am Samerberg die letzte Warmzeit - nach der im Bereich der alpinen Vereisung üblichen Nomenklatur also die Riß/ Würm-Warmzeit - und das Frühwürm nachgewiesen. $\mathrm{Zu}$ diesem Ergebnis hatten schon der geologische Befund und der überregionale Vergleich der interglazialen Vegetationsentwicklung geführt.

\section{Diskussion}

Aus dem Vergleich der nunmehr fünf umfassenden jungpleistozänen Vegetationsabfolgen ergibt sich, daß die interglaziale Vegetationsentwicklung sowohl im $\mathrm{S}$ als auch im N Mitteleuropas eine ungefähr gleiche Höhe erreichte. Während der Frühwürm-Interstadiale war dies offensichtlich nicht der Fall; der Vegetationsgradient verlief steiler. Die anspruchsvollste Vegetation kennen wir im Frühwürm vom Südwestfuß der Vogesen. Vom Alpennordrand ist eine relativ anspruchsvolle, aber montan geprägte Flora bekannt, und 
die anspruchsloseste Vegetation fand sich - wie zu erwarten - in Norddeutschland. Dieser Befund läßt auf die Existenz einer ausgeprägten Vegetationszonierung während des Frühwürms schließen, wie man sie bisher nicht erwartet hat.

Es nimmt deshalb nicht wunder, daß WoILlard (1975) die Waldzeiten St. Germain I und II wegen ihrer anspruchsvollen Vegetation (Carpinus!) nicht mit den FrühwürmInterstadialen gleichsetzte, obwohl sie das Interglazial im Liegenden Eem nennt. WoILLard bezeichnete St. Germain I und II als Interglaziale und schob diese als zusätzliche, bis dahin unbekannte Warmzeiten zwischen Eem-Interglazial und Würm-Kaltzeit ein. Dies geschah, obwohl dann in Grande Pile, dessen Pollendiagramme bis in das Postglazial reichen, bei einer derartigen Zuordnung ein überzeugender Nachweis von Frühwürm-Interstadialen nicht mehr möglich ist, obwohl bekannt war, daß es im Frühwürm in SchleswigHolstein und Jütland zu einer Bewaldung mit Kiefer und Fichte kam und obwohl in den Niederlanden, z. B. im Frühwürm von Amersfoort ansehnliche Mengen von QuercusPollen nachgewiesen worden waren (ZAGWIJN 1961).

Es ist demnach nicht zwingend notwendig, zusätzliche Warmzeiten zwischen Eem und Holozän einzuschieben. Damit wird auch der Folgerung WoIllard’s, Eem und Riß/Würm seien nicht zeitgleiche Warmzeiten, der Boden entzogen. Allerdings entsteht ein nomenklatorisches Problem, denn - wie gezeigt werden konnte - kann eine Flora interglazialen Charakters im S zeitgleich sein mit einer Flora interstadialen Charakters im N.

Das Amersfoort-Interstadial stellt offenbar einen Teil des hier als zweigeteilt bezeichneten ältesten Interstadials dar (vgl. Menke 1976, Menke \& BeHre 1973).

Kenntnis von drei in einem einzigen ungestörten Profil aufeinanderfolgenden interstadialen Waldzeiten haben wir bisher nur von Tenagi Philippon in NW-Griechenland und vom Samerberg. Bei Tenagi Philippon sind nach Wijmstra (1969) aber Äquivalente des Amersfoort-, Brørup- und Odderade-Interstadials nachgewiesen worden, von denen die beiden ältesten von WoIllard (1978) mit St. Germain I A und I C, also mit dem zweigeteilten ältesten Frühwürm-Interstadial nördlich der Alpen, parallelisiert werden, was sicherlich richtig ist, und das dritte dem Odderade gleichgesetzt wird. Für das dritte, nur fragmentarisch überlieferte Interstadial vom Samerberg gibt es aber anderswo noch kein Äquivalent.

Zum Schluß seien einige Bemerkungen zu den süddeutschen Interglazial- und Interstadialvorkommen angefügt.

Keines der bekannten Vorkommen, auch nicht die vom Wurzacher Becken (German, Filzer u. a. 1968) und vom Pfefferbichl (FILZER 1967), scheint eine so umfangreiche Vegetationsabfolge zu umfassen wie die Seeablagerungen vom Samerberg. Die meisten der als riß/würmzeitlich angesehenen Vegetationsabfolgen lassen sich mit der vom Samerberg parallelisieren; am besten die Abfolgen von den zueinander am nächsten gelegenen Vorkommen Zeifen (in der Nähe des Waginger Sees, Jung, Beug \& Denm 1972), Eurach südlich des Starnberger Sees (BEug 1973) und auch Mondsee (KLAus 1975). Selbstverständlich weisen die Pollendiagramme von diesen Untersuchungspunkten Unterschiede auf; aber diese spiegeln - wie man dem Vergleich der heutigen Klimadaten leicht entnehmen kann - sehr genau die klimatischen Unterschiede an diesen Untersuchungspunkten wider. So herrschen im tief gelegenen, kontinentalen Zeifen die Arten der Tieflagen vor, während am Samerberg die montanen Arten — seiner Lage am Alpenrand entsprechend - stärker ins Gewicht fallen. Eurach, das ebenso hoch liegt wie der Samerberg, aber weit vom Alpenrand entfernt ist, nimmt eine zwischen Zeifen und Samerberg vermittelnde Stellung ein.

Schwieriger ist die Parallelisierung der verschiedenen Interstadialvorkommen und von Endabschnitten von Interglazialen, die bekanntlich Interstadialen ähneln. Ursache hier- 
für ist die Tatsache, daß die Vegetationsentwicklung der Interstadiale nur wenige charakteristische Unterschiede aufweist und oft nur bruchstückhaft überliefert ist. Solche Vorkommen werden wahrscheinlich bezüglich ihrer Datierung noch lange Zeit Probleme aufwerfen und deshalb für die Gliederung des Pleistozäns nicht herangezogen werden können.

Lange Sequenzen - wie die vom Samerberg oder Grande Pile - bieten zur Zeit immer noch die besten Ansatzpunkte für eine Gliederung, wenn nicht des Pleistozäns, so doch einzelner Abschnitte desselben.

\section{Schriftenverzeichnis}

Averdieck, F.-R. (1967): Die Vegetationsentwicklung des Eem-Interglazials und der FrühwürmInterstadiale von Odderade/Schleswig-Holstein. - Fundamenta, Monographien zur Urgeschichte B/2, 101-105, 3 Abb., 2 Tab., 4 Tafeln; Köln (Böhlau).

BEug, H.-J. (1973): Die Bedeutung der interglazialen Ablagerungen von Zeifen und Eurach (Oberbayern, BRD) für die Vegetationsgeschichte der Eem-Warmzeit am Nordrand der Alpen. - In: Grichuk, V. P. (ed.): Palynology of Pleistocene and Pliocene. Proceedings of the IIIrd International Palynological Conference: 7-13, 1 Abb., 1 Tab.; Moskau (NAUKA).

ErD, K. (1973): Pollenanalytische Gliederung des Pleistozäns der Deutschen Demokratischen Republik. - Z. geol. Wiss. 1: 1087-1103, 8 Abb., 1 Tab.; Berlin.

Filzer, P. (1967): Das Interglazial Riss-Würm vom Pfefferbichl bei Buching im Allgäu. - Vorzeit, 1967, 1-4: 3-18, 9 Abb., 2 Tab.; Singen am Hohentwiel (Hegau).

German, R., Filzer, P., Dehm, R., Freude, H. Jung, W. \& Witt, W. (1968): Ergebnisse der wissenschaftlichen Kernbohrung Wurzacher Becken 1 (DFG). - Jh. Ver. vaterl. Naturkde. Württemberg, 123: 3-68, 6 Abb., 3 Tab.; Stuttgart.

GrüGER, E.: Spätriß, Riß/Würm und Frühwürm am Samerberg in Oberbayern - ein vegetationsgeschichtlicher Beitrag zur Gliederung des Jungpleistozäns. - Geol. Bavaria, 79: München. - [Im Druck].

Jung, W., Beug, H.-J. \& Deнm, R. (1972): Das Riß/Würm-Interglazial von Zeifen, Landkreis Laufen a. d. Salzach. - Bayer. Akad. Wiss., Math.-Nat. Kl., Abh., N. F., 151: 1-131, 15 Abb., 7 Taf.; München.

Klaus, W. (1975): Das Mondsee-Interglazial, ein neuer Florenfundpunkt der Ostalpen. - Jb. OOO. Musealvereines, 120: 315-344, 5 Abb., 5 Taf.; Linz.

Menke, B. (1976): Neue Ergebnisse zur Stratigraphie und Landschaftsentwicklung im Jungpleistozän Westholsteins. - Eiszeitalter u. Gegenwart, 27: 53-68, 1 Abb., 3 Tab.; Öhringen/ Württ.

- \& Behre, K.-E. (1973): State of research on the Quaternary of the Federal Republic of Germany. 2. History of vegetation and biostratigraphy. - Eiszeitalter u. Gegenwart, 23/24: 251-267, 1 Abb., 1 Tab.; Ơhringen/Württ.

Rabien, I. (1953): Die Vegetationsentwicklung des Interglazials von Wallensen in der Hilsmulde. - Eiszeitalter u. Gegenwart, 3: 96-127, 11 Abb., 11 Abb., 1 Tab.; O'hringen/Württ.

Schneekloth, H. (1966): Ergebnisse weiterer Unteruchungen an den interstadialen Ablagerungen der Weichsel-Eiszeit in Oerel/Krs. Bremervörde. - Z. dt. geol. Ges., 116: 773-796, 6 Abb., 2 Tab.; Hannover.

Wijmstra, T. A. (1969): Palynology of the first 30 meters of a $120 \mathrm{~m}$ deep section in northern Greece. - Acta Bot. Neerl., 18: 511-527, 5 Abb., 1 Taf.; Amsterdam.

Woillard, G. (1975): Recherches palynologiques sur le pleistocene dans l'est de la Belgique et dans les Vosges Lorraines. - Acta Geogr. Lovaniensia, 14: 118 p., 7 Abb., 8 Tab., 40 Taf.; Louvain-La-Neuve.

Woillard, G. M. (1978): Grande Pile peat bog: a continuous pollen record for the last 140,000 years. - Quaternary Research, 9: 1-21, 5 Abb., 1 Tab.; New York.

ZAGwiJn, W. H. (1961): Vegetation, climate and radiocarbon datings in the late pleistocene of the Netherlands. 1. Eemian and early Weichselian. - Memoirs Geol. Found. Netherlands, N. S., 14: 15-45, 14 Abb., 2 Tab., 8 Taf.; Harlem. 